$\begin{array}{cc}\text { PRAMANA } & \text { (c) Indian Academy of Sciences } \\ \begin{array}{c}\text { journal of } \\ \text { physics }\end{array} & \begin{array}{r}\text { Vol. 53, No. } 6 \\ \text { December } 1999 \\ \text { pp. } 1-11\end{array}\end{array}$

\title{
Aging, rejuvenation and memory phenomena in spin glasses
}

\author{
V. Dupuis, F. Bert, J.-P. Bouchaud, J. Hammann, F. Ladieu, D. Parker and E. \\ Vincent \\ Service de Physique de l'Etat Condensé (CNRS URA 2464), DSM/DRECAM, CEA \\ Saclay, 91191 Gif sur Yvette - France
}

\begin{abstract}
In this paper, we review several important features of the out-of-equilibrium dynamics of spin glasses. Starting with the simplest experiments, we discuss the scaling laws used to describe the isothermal aging observed in spin glasses after a quench down to the low temperature phase. We report in particular new results on the sub-aging behaviour of spin glasses. We then discuss the rejuvenation and memory effects observed when a spin glass is submitted to temperature variations during aging, from the point of view of both energy landscape pictures and of real space pictures. We highlight the fact that both approaches point out the necessity of hierarchical processes involved in aging. Finally, we report an investigation of the effect of small temperature variations on aging in spin glass samples with various anisotropies which indicates that this hierarchy depends on the spin anisotropy.
\end{abstract}

Keywords. spin glass, relaxation, aging, rejuvenation, memory

PACS Nos 75.10.Nr, 75.40.Gb, 75.50.Lk

\section{Introduction}

Spin glasses are magnetic systems in which the interactions are disordered and frustrated, due to a random dilution of magnetic ions. Below their glass temperature $T_{g}$, their dynamic response to a small magnetic excitation is slow, and in addition depends on the time spent below $T_{g}$ ('aging') [1,2]. In contrast with structural and polymer glasses, aging in spin glasses is hardly influenced by the cooling rate. On the contrary, each cooling step leads to a restart of dissipation processes ('rejuvenation'), while the memory of previous agings achieved at different temperatures during cooling can be retrieved during re-heating [3,4]. It is possible to store independently the memory of several isothermal agings, which corresponds to imprinting the trace of various spin arrangements at well separated length scales that are selected by temperature $[5,6]$.

In this paper, we first recall in section 2 some basic features of isothermal aging in spin glasses. We discuss in particular the scaling laws used to describe the response of a spin glass to a field variation and report new results which raise the question of the link between the cooling time and the sub-aging behaviour observed in several 
spin glasses. We then examine in section 3 the effect of temperature variations on aging which reveals striking rejuvenation and memory effects and discuss these effects in terms of energy landscape/real space pictures. Section 4 is devoted to a comparison between an Ising spin glass and several Heisenberg spin glasses with various degrees of random anisotropy, which shows that the aging properties are markedly different in these systems, the memory effect being more pronounced in the Heisenberg case $[7,8]$. These last results underline the role of anisotropy in the nature of the spin-glass phase with Heisenberg spins and are discussed in the context of a crossover expression for the relationship between time and length scales in an aging spin glass.

\section{Isothermal aging in spin glasses}

\subsection{Basics features and scaling laws}

A large variety of systems exhibit slow glassy dynamics and aging effects, i.e. history dependent properties. In all these systems, the response to an external excitation depends on the time $t_{w}$ (waiting time) that the system has spent in the low temperature phase before the excitation is applied. In most cases, the response function can be typically expressed as the sum of a fast stationary part and a slow aging contribution which roughly scales as $t / t_{w}$ (Figure 1a) $[1,2]$ :

$$
R\left(t_{w}+t, t_{w}\right)=R_{s}(t)+R_{a}\left(t / t_{w}\right)
$$

where $t_{w}$ is the time elapsed at the measurement temperature $T_{m}$ since the system has been cooled down below its glass temperature $T_{g}$, and $t$ is the time elapsed since the excitation has been applied. This scaling means that the effective relaxation time of the system below its freezing temperature is mainly set by its age $t_{w}+$ $t$. It is observed not only in the response to a field variation but also in the $a c$ susceptibility (harmonic response) which obeys an equivalent $\omega t_{w}$ scaling, where $\omega$ is the frequency of the $a c$ field [1].

The $t / t_{w}$ scaling is however only approximate. It appears that a more precise scaling can be obtained by assuming that the effective relaxation time goes as the full age $\left(t+t_{w}\right)$ to a certain power $\mu$ with $\mu<1$ (Figure 1b). This leads to an effective scaling variable $\xi=\lambda / t_{w}^{\mu}=1 /(1-\mu)\left[\left(t+t_{w}\right)^{1-\mu}-t_{w}^{1-\mu}\right]($ see $[9,1])$. $\mu<1$ means that the effective relaxation time grows more slowly than the age of the system, this situation is thus called sub-aging. Many examples of out-of-equilibrium systems such as spin glasses, glassy polymers, microgel pastes and other soft glassy materials, exhibit this sub-aging behaviour. In all these cases, the exponent is smaller than 1, but remains close to 1 (of order 0.8-0.9) in a rather large domain of temperatures $\left(0.4<T / T_{g}<0.9\right)$. Such a behaviour is predicted in simple trap models [10], in some mean-field models of spin-glasses [2] and in a recent extended droplet model $[11,12]$. 

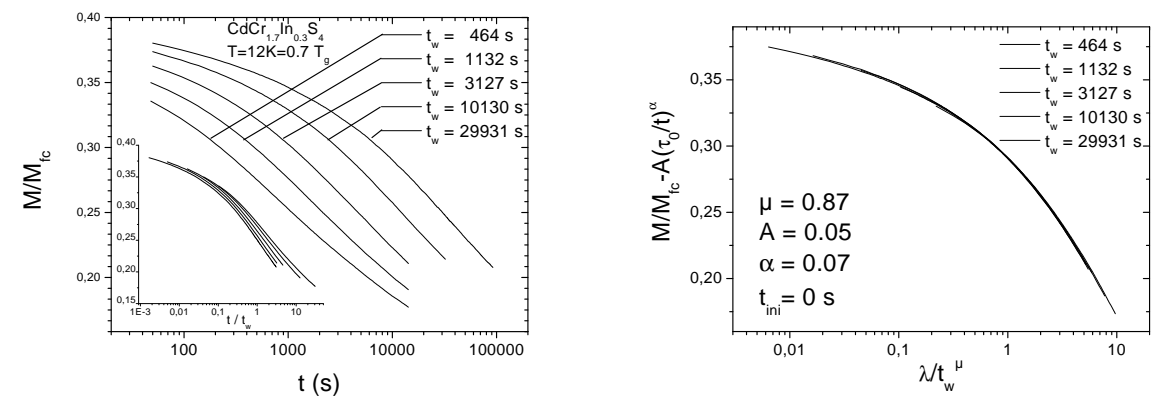

Figure 1. (left) thermoremanent magnetization relaxations at $0.7 T_{g}$ on the thiospinel $\mathrm{CdCr}_{1.7} \mathrm{In}_{0.3} \mathrm{~S}_{4}$ spin glass $\left(T_{g}=16.7 \mathrm{~K}\right)$; (right) scaling of the previous relaxation curves (see text for details).

\subsection{Intrinsic or extrinsic departure from full $t / t_{w}$ scaling}

The important question of whether the experimental value of $\mu$ smaller than 1 may not be due to experimental artefacts is however far from being settled and has given rise to much interest recently [13]. There are three main experimental limitations which may have a crucial effect on the results: the size limitations of the samples, the application of finite excitations, the long time needed to cool the sample down to the measurement temperature.

In a finite system composed of many subsystems (which could be grains in polycristalline samples or powder), one can expect that the ergodic times needed to explore the whole configuration space of each subsystem remain also finite. In that case aging will be interrupted at some ergodic time and the exponent $\mu$ will decrease and eventually go to zero as the ergodic time is reached in an increasing number of subsystems. This is a natural mechanism for sub-aging which has been used in $[14,15]$ to estimate, from the observed values of $\mu$, the cut-off of the barrier height distribution or that of the size distribution of the considered samples. However no experimental proof of the effect could yet be given.

The amplitude of the applied magnetic field is obviously also very important. Usually, only the minimum necessary excitations are used in an aging investigation and care is taken to ensure the response remains in the linear regime. However it is very difficult to understand in which way and how much the system is perturbed. A systematic study of the field effect was performed in spin glasses [16] and showed that $\mu$ indeed decreases as the excitation field is increased. However, it seems to saturate at very low fields and stays at a value smaller than 1 as the field is reduced by large factors. In the case of the thiospinel compound, $\mu$ saturates around 0.85 below 10 Gauss down to the smallest field probed $\left(10^{-3}\right.$ Gauss) [17].

Recently some detailed investigations were devoted to the problem of the cooling time and the importance of the initial conditions in the measurement of the thermoremanent magnetisation (TRM) in spin glasses $[18,13,19]$. Different cooling procedures were used and their effects on the TRM were considered. In the quoted 
investigations, three different cooling protocols were used: (A) Direct cooling from above $T_{g}$ to the measuring temperature $T_{m}$ (usual procedure); (B) Fast cooling from above $T_{g}$ down to a temperature smaller than $T_{m}$ and heating back up to $T_{m}$; (C) Cooling down to a temperature larger than $T_{m}$ and waiting a certain time before the final cooling step down to $T_{m}$.

Some differences have been found using these various initial conditions. In [13] it was argued that a full aging $(\mu=1)$ could almost be obtained in a $\mathrm{Cu}: \mathrm{Mn}_{6 \%}$ spin glass sample with protocol (B). In [19], the difference between the two protocols (A) and (B) in a $\mathrm{Ag}: \mathrm{Mn}_{11 \%}$ sample is only to reduce the initial age of the system for the shorter cooling time (protocol (B)).

\subsection{Revisiting the link between cooling time and sub-aging}

In order to clarify the situation, we have performed new experiments using protocols (A),(B),(C) described above, to check the effects of different initial conditions on the scaling behaviour of the response function of three well characterized spin glasses.

In order to precisely estimate the real cooling times associated with a given protocol, we recorded the field cooled magnetization, which actually slightly depends on temperature and can thus be used as a thermometer giving the true temperature of the sample. In all cases, TRM measurements made just after the temperature stabilization at $T_{m}$ show a maximum of $\mathrm{dM} / \mathrm{d}(\log \mathrm{t})$ at a time of the order of the real cooling time. In other words the cooling time in these experiments corresponds to some equivalent initial waiting time $t_{i n i}$ at $T_{m}$.

Fig. 2a shows a scaling of several TRM curves measured according to protocol (B) for various waiting times with the Heisenberg-like thiospinel $\mathrm{CdCr}_{1.7} \mathrm{In}_{0.3} \mathrm{~S}_{4}$ spin glass. The same sub-aging behaviour as in protocol (A) (Fig. 1b) can be observed with a slightly decreased value of the exponent $\mu(\mu=0.83$ here, compared to $\mu=0.87$ in protocol $(\mathrm{A})$ ). However an experiment made following protocol $(\mathrm{C})$, showed the same result $(\mu=0.87)$ as with the usual cooling protocol (A), even though the sample was kept for 1 hour at $T / T_{g}=0.94$ before being cooled down to the measuring temperature $T_{m} / T_{g}$ (Fig. 2b). Other experiments on a $\mathrm{Au}: \mathrm{Fe}_{8 \%}$ sample, another Heisenberg-like spin glass which is expected to behave similarly as the thiospinel [8], do not show any clear trend when slowing down the cooling procedure. The results are compatible with the thiospinel results, though less accurate due to a smaller signal, and point thus towards the same tiny cooling effects, if any.

We performed the same experiments on $\mathrm{Fe}_{0.5} \mathrm{Mn}_{0.5} \mathrm{TiO}_{3}$, an Ising spin glass in which stronger cooling effects can be expected [7]. The scaling of the TRM relaxation curves measured with fast (protocol (B)) and slow (protocol (C)) coolings is presented in Fig. 3. Fast cooling (3a) yields $\mu=0.84$ and $t_{i n i}=75 \mathrm{~s}$, with a good scaling. Slower cooling yields, as can be seen in 3b and 3c, (i) a downgrading of the scaling quality, as noted in [13] (ii) a change in the scaling parameters which can be as well accounted for by an increase of $t_{\text {ini }}$ or a decrease of $\mu$.

From this analysis, we conclude that there is no evidence that $\mu$ would go to 1 for a very fast cooling. Our results are compatible with the idea of an 'intrinsic' nature of the sub-aging behaviour in spin glasses. In numerical simulations of (Ising) spin 
glasses, in which cooling can be instantaneous, it has been shown [20] that the effect of cooling rate on the value of $\mu$ very quickly saturates. $\mu$ was found to evolve from a value slightly above 1 for infinite cooling rate to a value less than 1 when the cooling rate is of the order of $10^{-2}$ in microscopic units, and does not evolve significantly when the cooling is slower. Since a cooling rate of $10^{-2}$ is still very much faster than any experimentally achievable cooling rate, this scenario observed in numerical simulations may be a plausible explanation for sub-aging in real materials.
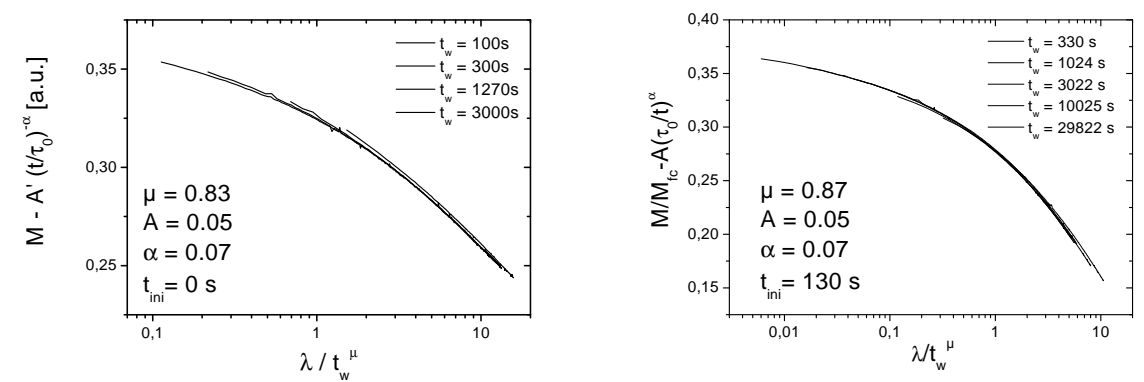

Figure 2. Scaling of thermoremanent magnetization relaxations measured on the $\mathrm{CdCr}_{1.7} \mathrm{In}_{0.3} \mathrm{~S}_{4}$ spin glass at $0.7 T_{g}$. (left) fast cooling (protocol (B)) (for this set of data, the exact vertical scale is unknown, but within the error bars A' corresponds to $\mathrm{A}=0.05$ ) ; (right) slow cooling (protocol $(\mathrm{C})$ ) (see text for details).

\section{Memory effects and hierarchy of states/length scales}

In the previous section, we discussed what happens when one simply quenches a spin glass below its glass transition temperature $T_{g}$ and lets it evolve at constant temperature. Other protocols may be imagined in which one can probe the effects of temperature variations during aging and see, for example, how far heating slightly an aging spin glass can help it to reach its equilibrium $[3,4]$.

Figure 4 shows the result of such a protocol on the imaginary part $\chi^{\prime \prime}$ of the $a c$ susceptibility measured at $0.1 \mathrm{~Hz}$ on the $\mathrm{CdCr}_{1.7} \mathrm{In}_{0.3} \mathrm{~S}_{4}$ spin glass. In this experiment, the sample was step-cooled through its glass transition temperature $T_{g}=16.7 \mathrm{~K}$ down to $5 \mathrm{~K}$ (each plateau at constant temperature lasts approximatively $30 \mathrm{~min}$ ) and reheated then continuously up to above $T_{g}$. During each stop at constant temperature, aging is directly evidenced as a downward relaxation of $\chi^{\prime \prime}$. Looking carefully at the figure, we see that further cooling seems to rejuvenate the system as the susceptibility rises back: this is the rejuvenation effect. However, when heated back continuously (full symbols), $\chi^{\prime \prime}$ shows marked dips at the plateau temperatures where the sample was allowed to age, revealing that the system has kept the memory of each stop that occurred during the cooling. 

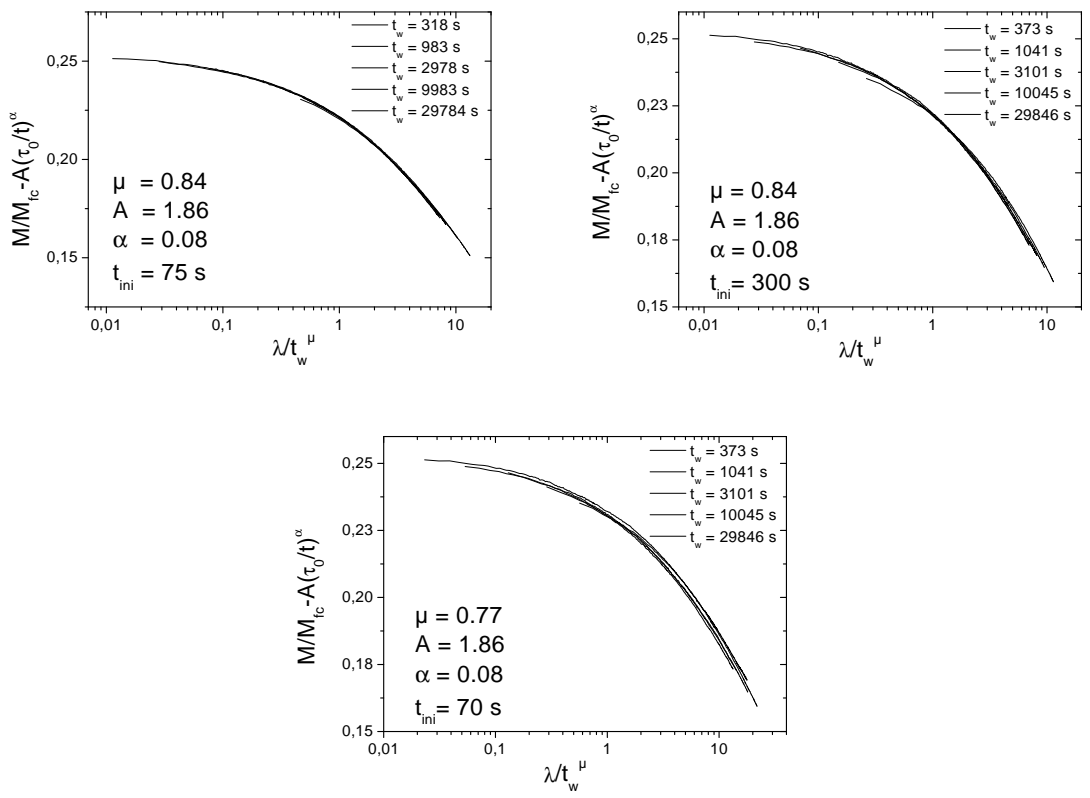

Figure 3. Scaling of thermoremanent magnetization relaxations measured on the $\mathrm{Fe}_{0.5} \mathrm{Mn}_{0.5} \mathrm{TiO}_{3}$ Ising spin glass at $0.7 T_{g}$ : (top left) fast cooling (protocol (B)) ; (top right) slow cooling (protocol (C)), scaling with fixed $\mu$; (bottom) slow cooling (protocol (C), same data), but with fixed $t_{i n i}$.

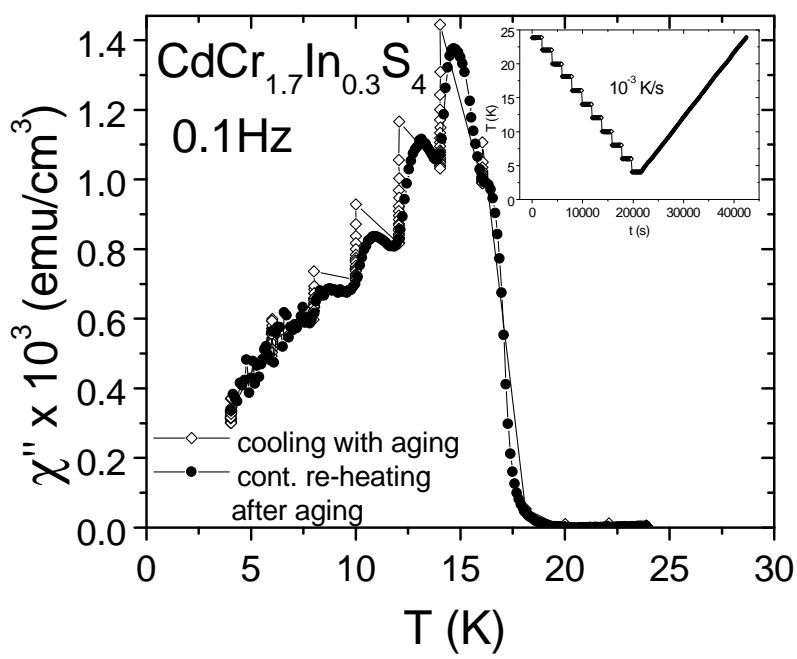

Figure 4. Series of dips imprinted on the ac susceptibility by successive stops at different temperatures while the system is cooled. 
Energy landscape pictures: These striking rejuvenation and memory effects can be interpreted in terms of a hierarchical organization of the metastable states in which the system evolves during its search for equilibrium [3,1]. This picture, originally inspired from the mean field solution of the SK spin glass model, was further developed in the past years in several toy models such as the "traps on a tree' model [21] or the Sinai model (in which the hierarchy is put by hand) ( [22]). On the other hand, recent numerical simulations of the Generalized Random Energy Models [23] (roughly equivalent to the 'traps on a tree' model) proved unambiguously that the idea of a hierarchy of metastable states is able to capture the main features of experimentally observed aging.

Real space pictures: While energy landscape pictures are illuminating, one cannot avoid trying to interpret the dynamics in an aging spin glass in real space, that is in terms of spin configurations. It is now widely accepted that aging in spin glasses corresponds to a growth of a certain coherence length (which would be the equivalent of a kind of out-of-equilibrium correlation length) $[12,5]$. In a ferromagnet, for example, this coherence length is simply the size of the ferromagnetic domains and the aging observed after a quench just reflects the growth of these ferromagnetic domains. In spin glasses however, and contrary to ferromagnets where the domain growth is directly related to cooling rate (the longer the time spent at higher temperature, the larger the domains), aging is hardly influenced by the cooling rate, as evidenced in Fig. 4 where each cooling step seems to entirely rejuvenate the system.

A simple real space scenario which allows one to reconcile the idea of a growing coherence length with the observed rejuvenation and memory effects was discussed [5]. It was noticed that a thermally activated dynamic over energy barriers $B \sim \Upsilon(T) \ell^{\psi}$ that vanish at $T_{g}$ as $\Upsilon(T) \sim\left(T-T_{g}\right)^{\psi \nu}$ (B is the barrier to overcome in order to flip a cluster of spins of typical size $\ell$ ) naturally leads to a hierarchy of the length scales probed in experiments. More precisely, and as described in [5], the temperature $T$ at which aging is observed sets the length scale $\ell^{*}(T)$ at play in aging (often called active length scale). Larger length scales $\left(\ell>\ell^{*}(T)\right)$ are frozen while smaller length scales $\left(\ell<\ell^{*}(T)\right)$ are fully equilibrated. Decreasing the temperature from $T$ to $T-\Delta T$ reduces significantly this typical length scale as $\ell^{*}(T-\Delta T)<\ell^{*}(T)$. This has two important consequences. Firstly the structure previously reached at length scale $\ell^{*}(T)$ freezes since the time needed to reconform details of the structure at this length scale has grown astronomically large. This will allow the frozen structure to be retrieved by heating back the system (memory effect). Secondly, the length scales $\ell$ such that $\ell^{*}(T-\Delta T)<\ell<\ell^{*}(T)$, which were equilibrated at $T$, are no longer equilibrated at $T-\Delta T$ due to the change with temperature of the associated Boltzmann weights. They start to age giving rise to the rejuvenation effect. This scenario, which naturally leads to a hierarchy of embedded active length scales, is an appealing transcription of the hierarchical energy landscape pictures. An alternative scenario, based on 'temperature chaos', has been actively promoted in [26]. Although the mechanism leading to rejuvenation is different, this scenario also requires a rapid separation of time scales as the temperature is reduced to preserve the memory.

Pramana - J. Phys., Vol. 53, No. 6, December 1999 


\section{Spin glass dynamics, anisotropy and growth of the coherence length}

In order to go deeper into the understanding of the out-of-equilibrium dynamics of spin glasses and in particular the rejuvenation and memory effects discussed above, we recently performed a systematic study of the effect of temperature variations on the aging dynamics of various spin glasses with different degrees of anisotropy ranging from Ising to Heisenberg [7,8].

Temperature cycling experiments were performed in a monocrystalline $\mathrm{Fe}_{0.5} \mathrm{Mn}_{0.5} \mathrm{TiO}_{3}$ Ising [24] sample \#1 $\left(T_{g}=20.7 K\right)$ and in Heisenberg SGs with decreasing random anisotropy [25]: an amorphous alloy $\left(\mathrm{Fe}_{0.1} \mathrm{Ni}_{0.9}\right)_{75} \mathrm{P}_{16} \mathrm{~B}_{6} \mathrm{Al}_{3} \# 2$ $\left(T_{g}=13.4 K\right)$, a diluted magnetic alloy $\mathrm{Au}: \mathrm{Fe}_{8 \%} \# 3\left(T_{g}=23.9 \mathrm{~K}\right)$, an insulating thiospinel $\mathrm{CdCr}_{1.7} \mathrm{In}_{0.3} \mathrm{~S}_{4} \# 4\left(T_{g}=16.7 \mathrm{~K}\right)$ and the canonical Ag: $\mathrm{Mn}_{2.7 \%} \mathrm{SG} \# 5$ $\left(T_{g}=10.4 K\right)$ (data from $\left.[27]\right)$.

In these experiments, an isothermal TRM procedure allows one to first define a set of reference curves that depend on the waiting time (such as the one in Fig. 1), and to which we will compare the relaxation curves obtained after more complicated histories. In the temperature cycle experiments, the sample is cooled to a temperature $T<T_{g}$, kept at $T$ for a time $t_{1}=500 \mathrm{~s}$, submitted to long wait at $T-\Delta T$ during $t_{2}=9000$ s and finally re-heated to $T$ for another short time $t_{3}=t_{1}$. The magnetic field is then switched off and the TRM is recorded. For each 'cycled' TRM curve, an effective waiting time $t_{2}^{e f f}$ is defined such as to superimpose the obtained TRM curve with that of the purely isothermal TRM recorded at temperature $T$, with waiting time $t_{w}=t_{1}+t_{2}^{\text {eff }}(T, \Delta T)+t_{3}$. For $\Delta T=0$, the effective waiting time equals the actual waiting time, but becomes smaller for $\Delta T>0$ : $t_{2}^{e f f}(T, \Delta T)<t_{2}$ characterizes the effect at $T$ of aging at $T-\Delta T$. The results for the different SGs and for two different temperatures, $T / T_{g} \simeq 0.72$ and $T / T_{g} \simeq 0.85$, are plotted in Fig. 5 (for sample \#5, a similar procedure, with $t_{1}=t_{3}=0$, was applied [27]).

The results of Fig. 5 which correspond to small $\Delta T$ allow us to probe the separation of length scale discussed in the previous section [5]. Indeed, when considering two temperatures $T$ and $T-\delta T$ such that $\ell^{*}(T-\delta T) \sim \ell^{*}(T)$, rejuvenation and memory effects are no longer observed and aging tends to accumulate from one temperature to another, which we described here in terms of an effective time $t_{2}^{e f f}$. The stronger the decrease of this effective time with increasing $\delta T s$, the sharper the separation of active length scales. Fig. 5 thus shows that the separation of length scales becomes sharper and sharper when going from the Ising sample towards Heisenberg spin glasses with weaker and weaker anisotropy. Our results single out the role of the spin anisotropy in the nature of the Heisenberg spin glass phase.

A purely activated scenario over constant height barriers would correspond to a single straight line for all samples in Fig. 5 [8]. In order to account for the different observed behaviours, we introduced a crossover expression for the relationship between time and length scales

$$
t_{w}=\tau_{0} \ell^{z} \exp \left(\Delta(T) \ell^{\psi} / T\right)
$$

In this expression, which is simply an Arrhenius law with a temperature-dependent barrier, the usual attempt time $\tau_{0}$ is replaced by a renormalized attempt time $\tau_{0} \ell^{z}$

where $z$ is the dynamic critical exponent. This modification has the advantage of 


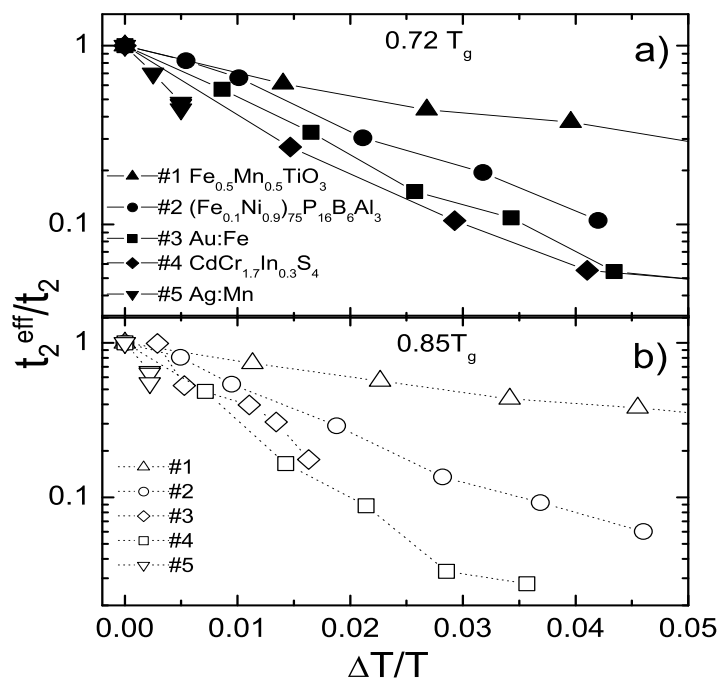

Figure 5. Effective waiting times deduced from the temperature cycle experiments at $T \simeq 0.72 T_{g}$ (a, filled symbols) and $T \simeq 0.85 T_{g}$ (b, open symbols). The SG's are labelled from \#1 to \#5 by order of decreasing anisotropy.

giving the expected form for the critical dynamics close to $T_{g}$, where due to the vanishing of the barrier height $\Delta(T)=\Upsilon_{0}\left(1-T / T_{g}\right)^{\psi \nu}$ the exponential term in Eq. (2) tends to 1 . In [8] the above expression was successfully used to explain consistently both temperature cycling experiments and field variation experiments. This in turn gave, for each sample, estimates of the barrier exponents $\psi$ and the energy scales $\Upsilon_{0}$ of the barriers which are consistent with a sharper separation of the active length scales in Heisenberg-like spin glasses than in the Ising case (see [8] for details). The same trend has recently been observed in large scale simulations of Ising and Heisenberg spin glasses [28].

Finally, let us mention an issue that concerns the value of the individual spin flip time $\tau_{0}$ (arising, e.g., in Eq. (2)), and that is usually assumed to be microscopic, of the order of $10^{-12}$ seconds. This assumption is very important since it means, as mentioned above, that typical experimental cooling rates are extremely slow when compared with $\tau_{0}$. However, it might well be that the flip of a single spin is already slowed down by its environment (interactions, local anisotropy...). In the Ising case, this slowing down might be somewhat large, and could significantly renormalise the value of $\tau_{0}$, bringing it much closer (especially at low temperatures) to the experimental time window. This could significantly affect the precise values of the parameters involved in Eq. (2). 


\section{Conclusion}

In this paper, we have briefly reviewed several important features of the slow out-ofequilibrium dynamics of spin glasses. We have first discussed the scaling laws that govern the isothermal aging of the response function of spin glasses, and showed experiments which suggest that the observed sub-aging behaviour may be intrinsic, although when compared to a similar effect observed in numerical simulations, laboratory cooling rates might still be too slow to conclude. We have then discussed the interpretation of the rejuvenation and memory effects observed in spin glasses submitted to temperature variations during their aging. In both energy landscape and real space pictures, the need for a hierarchy of state/length scales seems unavoidable for the description of such effects. This strong separation of length scales probed at different temperatures is also needed in the 'temperature chaos' interpretation of these rejuvenation/memory effects (see [26] for a review and detailed arguments supporting this point of view, and [29] for very recent numerical simulations challenging this interpretation).

We have reported experiments probing the effect of very small temperature variations in spin glass samples with various degrees of anisotropy. The main results of this last investigation are (a) a clear finger-print of a dynamical crossover between a critical regime and an activated regime in spin-glasses, with energy barriers vanishing at $T_{c}$ and (b) a trend to a sharper separation of active length scales with temperature in Heisenberg-like samples.

This work was supported in part by the European Community's Human Potential Programme (contract HPRN-CT-2002-00307 referred to as DYGLAGEMEM).

\section{References}

[1] E. Vincent, J. Hammann, M. Ocio, J.-P. Bouchaud and L.F. Cugliandolo, in Complex Behaviour of Glassy Systems, Springer Verlag Lecture Notes in Physics Vol. 492, M. Rubi editor, pp. 184-219 (preprint cond-mat/9607224), and references therein.

[2] J.P. Bouchaud, L.F. Cugliandolo, J. Kurchan, M. Mézard, in Spin Glasses and Random Fields, ed. A.P.Young, (World Scientific, Singapore, 1997), and references therein.

[3] Ph. Refregier, E. Vincent, J. Hammann and M. Ocio, J. Phys. (France) 48, 1533 (1987).

[4] K. Jonason, E. Vincent, J.P. Bouchaud, and P. Nordblad, Phys. Rev. Lett. 81, 3243 (1998); K. Jonason, P. Nordblad, E. Vincent, J. Hammann, and J.-P. Bouchaud, Eur. Phys. J. B 13, 99 (2000).

[5] J.-P. Bouchaud, V. Dupuis, J. Hammann and E. Vincent, Phys. Rev. B 65, 024439 (2002).

[6] S. Miyashita and E. Vincent, Eur. Phys. J. B 22, 203 (2001).

[7] V. Dupuis, E. Vincent, J.-P. Bouchaud, J. Hammann, A. Ito and H. Aruga Katori, Phys. Rev. B 64, 174204 (2001).

[8] F. Bert, V. Dupuis, E. Vincent, J. Hammann and J.-P. Bouchaud, Phys. Rev. Lett. 92, 167203 (2004). 
[9] L.C.E. Struik, Physical aging in amorphous polymers and other materials, (Elsevier, Houston, 1978).

[10] B. Rinn, P. Maass, J.P. Bouchaud, Phys.Rev.Lett. 84, 5403 (2000).

[11] H. Yoshino, K. Hukushima, H. Takayama, Phys.Rev.B 66, 064431 (2002).

[12] D.S. Fisher and D.A. Huse, Phys. Rev. B 38, 373 and 386 (1988).

13] G.F. Rodriguez, G.G. Kenning, R. Orbach, Phys.Rev.Lett. 91, 037203 (2003).

[14] J.-P. Bouchaud, E. Vincent, J. Hammann, J.Phys. I (France), 4, 139 (1994).

[15] Y.G. Joh, R. Orbach, G.G. Wood, J. Hammann, E. Vincent, J.Phys.Soc.Jpn. 69 Suppl. A, 215 (2000).

[16] E. Vincent, J.P. Bouchaud, D.S. Dean, J. Hammann, Phys.Rev. B 52, 1050 (1995).

[17] D. Hérisson and M. Ocio, Phys. Rev. Lett. 88, 257202 (2002); D. Hérisson and M. Ocio, Eur. Phys. J. B 40, 283 (2004).

[18] V.S. Zotev, G.F. Rodriguez, G.G. Kenning, R. Orbach, E. Vincent, J. Hammann, Phys. Rev. B 67, 184422 (2003).

[19] P. Nordblad, J.Phys.: Condens.Matter 16, S715 (2004).

[20] L. Berthier and J.-P. Bouchaud, Phys. Rev. B 66, 054404 (2002).

[21] J.-P. Bouchaud and D.S. Dean, J.Phys. I (France), 5, 265 (1995).

[22] M. Sales, J.P. Bouchaud, and F. Ritort, J.Phys. A: Math. Gen., 36, 665 (2003).

[23] M. Sasaki, V. Dupuis, J.-P. Bouchaud, and E. Vincent, Eur. Phys. J. B 29, 469 (2002).

[24] H. Aruga Katori and A. Ito, J. Phys. Soc. Jpn. 63, 3122 (1994).

[25] D. Petit, L. Fruchter and I. A. Campbell, Phys. Rev. B 65, 024439 (2001).

[26] P. E. Jönsson, R. Mathieu, P. Nordblad, H. Yoshino, H. Aruga Katori, A. Ito, Spin Glasses: A Ghost Story cond-mat/0307640.

[27] J. Hammann, M. Lederman, M. Ocio, R. Orbach and E. Vincent, Physica A 185, 278 (1992).

[28] L. Berthier and A.P. Young, Phys. Rev. B 69, 184423 (2004).

[29] S. Jimenez, V.Martin-Mayor, S. Perez-Gaviro, Rejuvenation and memory in model spin glasses in 3 and 4 dimensions, cond-mat/0406345. 